\title{
False recognition without intentional learning
}

\author{
MICHAEL D. DODD and COLIN M. MACLEOD \\ University of Toronto, Scarborough, Ontario, Canada
}

\begin{abstract}
Asked to memorize a list of semantically related words, participants often falsely recall or recognize a highly related semantic associate that has not been presented (the critical lure). Does this false memory phenomenon depend on intentional word reading and learning? In Experiment 1, participants performed a color identification task on distractor words from typical false memory lists. In Experiment 2, participants read the same words. In both experiments, the primary task was followed by a surprise recognition test for actually presented and unpresented words, including the critical lures. False alarms to critical lures were robust and quite equivalent across the two experiments. These results are consistent with an activation/monitoring account of false memory, in which processing of semantic associates can evoke false memories even when that processing is incidental.
\end{abstract}

Traditionally, memory research has emphasized accurate remembering, but memory errors can also be very informative about ongoing processes (Bartlett, 1932). Over the past decade, there has been increased interest in the occurrence of false memories in particular. Although initially spurred by the controversy over recovered memories of child abuse, most research on the topic has involved false memories for single words or concepts. The most common technique for investigating such errors has been the Deese/Roediger-McDermott (DRM) paradigm, which involves presenting a list of words, all related to a critical unpresented word (e.g., bed, pillow, and night are all related to sleep). Participants are instructed to memorize the presented words for a later test, ordinarily recall or recognition. Generally, false recall and recognition rates are fairly high for the unpresented critical lures (Deese, 1959; Roediger \& McDermott, 1995).

Several explanations have been advanced to account for false memories in the DRM paradigm, including the implicit associative response account (Underwood, 1965) and fuzzy trace theory (Brainerd \& Reyna, 2002). Recently, the activation/monitoring approach (see Roediger, Watson, McDermott, \& Gallo, 2001, for a review) has been gaining support. This approach holds that false memories arise during processing of DRM list words from either (1) conscious activation of critical lures via elaborative processes, or (2) automatic activation of critical

This research was supported by Discovery Grant A7459 from the Natural Sciences and Engineering Research Council of Canada. We appreciate the comments of Erin Sheard during the development of this project, and of the reviewers of the preceding version, Charles Brainerd, Kathleen McDermott, and John Seamon. Correspondence may be directed to the first author at the Department of Psychology, 100 St. George St., University of Toronto, Toronto, ON, M52 3G3 Canada (e-mail: michaeld@ psych.utoronto.ca) or to the second author, who is now at the Department of Psychology, University of Waterloo, Waterloo, ON, N2L 3G1 Canada (e-mail: cmacleod@watarts.uwaterloo.ca). lures via spreading semantic activation. Consequently, a key question concerns how words must be processed for false memories to be elicited. In particular, if false memories can be elicited automatically via spreading activation, then mere exposure to DRM list words ought to be sufficient to create a false memory. Researchers have reported findings that initially seem consistent with this claim.

Seamon, Luo, and Gallo (1998) had participants encode DRM lists, varying rate of presentation (2 sec, $250 \mathrm{msec}$, or $20 \mathrm{msec}$ per word). Under either full or divided attention, correct recognition of DRM list words decreased as presentation duration decreased, dropping below $50 \%$ at the $20-$ msec rate. False alarms to critical lures, however, remained generally robust regardless of presentation rate, decreasing slightly as duration decreased. Thus, false memory for critical lures occurred even when correct recognition of actual list words was very poor. Note that McDermott and Watson (2001) also used a $20-\mathrm{msec}$ presentation duration for list words, but their false recall rates were small relative to the false recognition rates obtained by Seamon et al. (1998).

In addition, investigators have explored the influence of various processing manipulations on the occurrence of false memories. Rhodes and Anastasi (2000) examined the effect of levels of processing (Craik \& Lockhart, 1972) on false recall by having participants either rate concreteness or count vowels as DRM list words were presented. Deeper processing yielded better overall memory and higher false recall of critical lures, but there were still false memories following shallow processing. Rhodes and Anastasi argued that their results were consistent with activation-based models of false memory, whereby deeper processing leads to greater activation of critical lures; shallow encoding presumably leads to less activation and, therefore, to reduced false memory. Toglia, Neuschatz, and Goodwin (1999) and Thapar and McDermott (2001) have reported similar effects of levels- 
of-processing manipulations, with veridical and false memories increasing in tandem.

Tussing and Greene (1997) also included deep (pleasantness ratings) and shallow (vowel counting, letter counting) processing tasks along with a number of other processing manipulations. Following encoding, participants engaged in a recognition test that was expected in all conditions except the three levels-of-processing conditions. They observed substantial false recognition in all encoding conditions, with lower rates in the incidental and mixed conditions. Contrary to Rhodes and Anastasi (2000), however, level of processing did not affect false recognition: There was no difference in false recognition between the pleasantness and counting conditions. ${ }^{1}$ Analogously, Read (1996) failed to obtain an effect of maintenance versus elaborative rehearsal on false recall. Nonetheless, the research strongly suggests that false memories can be elicited without semantic processing, in agreement with the activation/monitoring approach.

In each of the studies just described, robust false memory was observed despite differential processing of DRM words. In all cases, however, DRM words were intentionally processed - and usually intentionally memorizedin preparation for the memory test. Only Rhodes and Anastasi (2000) and Tussing and Greene (1997) employed surprise memory tests, but their participants still had to process the DRM words directly to perform the primary task (e.g., vowel counting) because the words were presented auditorily. What remains to be determined, then, is whether false memory can be elicited by the mere presentation of a DRM list, without any intention to read, memorize, or otherwise process the presented words. This is the principal purpose of our study.

To ensure unintentional (incidental) processing, in Experiment 1, we presented DRM list words as distractors in a Stroop-like color identification task, and then conducted a surprise recognition test. ${ }^{2}$ We contrasted this incidental word learning to the more standard intentional reading of the list words in Experiment 2. If false memories depend on intentional processing, then they should only occur in Experiment 2. In addition, we further examined the effect of levels of processing on false recognition, given the contrary findings about whether processing manipulations affect true and false memory similarly.

\section{EXPERIMENT 1}

In the Seamon et al. (1998) study, participants knew that a recognition test would follow and thus were intentionally reading and memorizing the list words, even at the short presentation duration. In the Rhodes and Anastasi (2000) and Tussing and Greene (1997) studies, although the recognition test was a surprise, list words had to be processed (albeit it in a shallow manner) to perform the primary task. Thus, processing was intentional in all of these prior studies. In contrast, in Experiment 1, the recognition test was a surprise, following an incidental study phase where participants ignored the words and identified their print colors. Because the primary task did not require reading the words, participants should not have attempted to process or memorize them during color identification.

It is well established that participants cannot avoid reading distractor words in a color naming task, although they do so automatically and unintentionally, as the classic Stroop effect demonstrates (Stroop, 1935; see C. M. MacLeod, 1991, for a review). It has been demonstrated that this automatic reading also occurs for noncolor words (e.g., C. M. MacLeod \& Dodd, 2001; Williams, Mathews, \& C. MacLeod, 1996). Of critical interest is the effect of unintentional reading processes on the false recognition of critical lures. If false recognition is attributable solely to intentional processing, then presenting DRM words under the guise of a color identification task should decrease or even eliminate false recognition. If, however, false recognition can be caused by activation of critical lures when related words are processed unintentionally, then substantial false recognition should be expected. The activation/monitoring approach predicts that mere exposure to DRM list words should suffice to produce false memories via activation of critical lures as related words are encountered in the list.

\section{Method}

Participants. Thirty-eight naive students from the University of Toronto at Scarborough took part individually in a 30-min session, receiving bonus points.

Apparatus. Experimental programs, written in QuickBASIC 4.5, used the Graves and Bradley (1991) routines to achieve millisecond timing. An IBM-compatible 486 computer displayed the stimuli on a Magnavox 15-in. color monitor. Participants identified colors using four response keys $(\mathrm{z}, \mathrm{x},>$, and / ) representing the four colors (red, blue, yellow, and green, respectively).

Materials. Distractor words for the color identification task consisted of the 12 strongest associates from 3 of the DRM lists (sleep, cold, and window) known to elicit high levels of false recognition (Stadler, Roediger, \& McDermott, 1999). List presentation order was counterbalanced. Words within each list were always presented in the same order-from strongest to weakest associate-following common practice with this procedure.

The recognition test consisted of 27 words: 12 actually presented words from the DRM lists (Words 2, 5, 8, and 11 from each list); 6 weakly related unpresented words (Words 13 and 14 from the norms for each list); 6 unrelated unpresented words from other DRM lists, and the 3 critical lures. All words were presented in standard DOS 80-character lowercase font on a black background.

Procedure. The participants sat about $30 \mathrm{~cm}$ from the monitor, responding via the keyboard. To become familiarized with the buttonpressing procedure for the color identification task, the participants initially performed 72 trials in which a row of asterisks appeared at the center of the screen in one of the four colors. ${ }^{3}$ They were instructed to indicate the color of the asterisks by pressing the corresponding key.

On each of the 36 color identification trials, a single word from one of the three DRM lists was presented at the center of the screen in one of the four colors. The participants were instructed to ignore the word and to indicate its print color as quickly and accurately as possible. Upon response, the word was replaced by a row of white asterisks, indicating that the next word would appear in $500 \mathrm{msec}$. Trials on which an incorrect keypress was made were omitted from analysis, as were 
trials having response times longer than 2,000 msec or shorter than $300 \mathrm{msec}$, although only true errors were counted as incorrect.

Finally, the participants were given a surprise recognition test for the words that they had been instructed to ignore during color identification. This test consisted of a random order of the 27 words described under Materials. Each word was presented in white at the center of the screen until the participant pressed " $\mathrm{z}$ " for new or "/" for old. The recognition test was self-paced and was followed by debriefing.

\section{Results and Discussion}

The data of four participants were discarded; two had language difficulties and two failed to follow instructions.

Color identification. Errors $(6.8 \%$ of all trials) were excluded from all response time analyses. Mean color identification time was $645 \mathrm{msec}(S D=105)$, typical for this task (see, e.g., C. M. MacLeod, 2000; C. M. MacLeod \& Dodd, 2001). This demonstrates that participants did not slow down to read or memorize the words intentionally, which was consistent with their not having expected the recognition test.

Recognition. We will first report the overall pattern of hits and false alarms. Then we will consider corrected recognition scores, taking into account false alarms made to both weakly related/unpresented words and unrelated/unpresented words. The proportions of "old" responses, representing correct recognition of actually presented DRM list words and false recognition of weakly related/unpresented words, unrelated/unpresented words, and critical lures, are presented in Table 1.

The mean proportions of "old" responses were analyzed using a one-way within-subjects analysis of variance (ANOVA). The effect of word type was significant $\left[F(3,99)=47.36, M S_{\mathrm{e}}=0.04, p<.001\right]$. Tukey HSD tests $(p<.05)$ showed that hits to actually presented words were significantly greater than false alarms to weakly related/unpresented words or to unrelated/unpresented words. There was no difference in false recognition rates between the latter two word types. Crucially, participants were very likely to false alarm to unstudied critical lures; indeed, they were considerably more likely to say "old" to a critical lure than to an actually presented word.

Table 1

Mean Probability of a "Yes" Response on the Recognition Test and Standard Deviation ( $S D)$ as a Function of Test Word Condition in Experiments 1 and 2

\begin{tabular}{lccccc}
\hline & \multicolumn{2}{c}{$\begin{array}{c}\text { Experiment 1 } \\
\text { Color Identification }\end{array}$} & & \multicolumn{2}{c}{$\begin{array}{c}\text { Experiment 2 } \\
\text { Word Reading }\end{array}$} \\
\cline { 2 - 3 } Word Type & $P$ ("Yes") & $S D$ & & $P$ ("Yes") & $S D$ \\
\hline Presented & .53 & .18 & & .83 & .13 \\
Critical lure & .70 & .31 & & .71 & .34 \\
Weakly related/unpresented & .25 & .16 & & .18 & .18 \\
Unrelated/unpresented & .19 & .20 & & .08 & .14 \\
Corrected critical lure & .47 & .33 & & .58 & .34 \\
\hline
\end{tabular}

Note-Scores represent hits for presented words, and false alarms for critical lures, weakly related/unpresented words, and unrelated/unpresented words. The final row reports corrected false recognition scores for critical lures, calculated as the false alarm rate to critical lures minus the average of the false alarm rates to the weakly related/unpresented and unrelated/unpresented conditions.
The relatively low hit rate for actually presented words and the quite low false alarm rates for weakly related/ unpresented and unrelated/unpresented words were anticipated. Indeed, these rates are almost identical to those of C. M. MacLeod and Dodd (2001), who also used a paradigm involving color identification study and a surprise recognition test. The high rate of false recognition for critical lures is intriguing given that participants were to ignore list words, but it is entirely consistent with the activation/monitoring approach of Roediger and colleagues, under which activation spreads to the critical lure.

Corrected recognition. Although false alarm rates for weakly related/unpresented words and unrelated/ unpresented words were quite low, they were not negligible. To take these into account, corrected false recognition scores for critical lures were determined by subtracting the false alarm rate for the average of weakly related/unpresented words and unrelated/unpresented words from the false alarm rate for critical lures. ${ }^{4}$ These values are presented in Table 1 . With this measure, participants still were as likely to falsely recognize a critical lure as they were to correctly recognize an actually presented word $[t(33)=1.15, p=.26]$.

The results suggest that unintentional processing of DRM list words is sufficient to produce substantial false recognition of critical lures. Before we proceed, however, one method-dependent explanation must be ruled out. Perhaps DRM words were not sufficiently encoded during color identification to support false recognition, with sufficient encoding arising only from the experiencing of the subset of list words presented in the recognition test. During the test, participants intentionally read presented words and made old/new decisions about them. If this test-driven account were correct, then false alarms to critical lures would be expected to increase as more words preceded the related critical lures at recognition.

A correlational analysis was performed to determine the probability of a false alarm to each critical lure as a function of the number of related words that preceded it in the recognition test. From zero to six related words could precede a critical lure: the four tested list targets plus the two weakly related/unpresented distractors. This analysis revealed no relation between the two variables $(r=.07, p=.50)$, indicating no greater likelihood of a false alarm to a critical lure preceded by more versus fewer related words. This suggests that false alarms to critical lures are not driven by direct experience of words on the recognition test; rather, they derive from processes occurring during the incidental color-naming study task. A related analysis showed that the probability of a false alarm to a critical lure did not vary as a function of whether the critical lure appeared in the first, second, or third portion of the test sequence $(F<1)$.

False alarms to critical lures occurred despite low recognition of actually presented words, as in Seamon et al. (1998), and despite shallow processing of the DRM list words, as in Tussing and Greene (1997). The present study is, however, the first to demonstrate that false memory can 
occur without attempted memorization or other intentional processing of DRM list words. Unintentional processing of related words is all that is necessary to produce substantial false recognition of critical lures. This contradicts any theory of false memory that attributes these errors to intentional, strategic processing of list words. It is thoroughly consistent, however, with the activation/monitoring approach, which maintains that false memories can be elicited via automatic spreading of semantic activation.

\section{EXPERIMENT 2}

In Experiment 2, participants were instructed to read the words and ignore their display colors. The participants were still unaware, however, of the upcoming recognition test. Our expectation was that switching from unintentional to intentional reading would increase the correct recognition of actually presented words and decrease the false recognition of weakly related/unpresented and unrelated/unpresented words. Of critical interest, however, was how the switch to intentional reading would affect the false recognition of critical lures. Would higher correct recognition for actually presented words promote higher false recognition for critical lures (as in Rhodes \& Anastasi, 2000), or would no change in false recognition be observed (as in Tussing \& Greene, 1997)?

\section{Method}

Participants. Thirty-four naive students from the same pool took part individually in a 30-min session.

Apparatus and Procedure. The apparatus and procedure were identical to those in Experiment 1, with a single modification: Participants now responded vocally to the words, ignoring their display colors. These oral responses were collected using a Realistic Highball-7 microphone interfaced to the computer through a Realistic amplifier.

\section{Results and Discussion}

Word reading. Mean word reading time was $600 \mathrm{msec}$ $(S D=77)$, which was again typical for this task (see C. M. MacLeod, 2000) and consistent with participants' not studying the words intentionally in anticipation of the recognition test.

Recognition. Proportions of "old" responses-representing correct recognition of actually presented DRM list words, as well as false recognition of weakly related/ unpresented words, unrelated/unpresented words, and critical lures-are presented in Table 1.

Mean proportions of "old" responses were analyzed using a one-way within-subjects ANOVA. The effect of word type was significant $\left[F(3,99)=124.58, M S_{\mathrm{e}}=\right.$ $0.04, p<.001]$. As expected, switching to intentional reading increased the correct recognition of actually presented words and decreased the false recognition of weakly related/unpresented and unrelated/unpresented words relative to Experiment 1. Impressively, however, false alarms to critical lures remained virtually identical to those in Experiment 1.

As in Experiment 1, Tukey HSD tests $(p<.05)$ showed that hits to actually presented words were significantly greater than false alarms to weakly related/unpresented words or to unrelated/unpresented words, which did not differ. The switch to intentional reading, however, did result in actually presented words' being correctly recognized more often than critical lures' being falsely recognized. False recognition of critical lures was still significantly greater than false recognition of all other word types.

Corrected recognition. As in Experiment 1, corrected false recognition scores for critical lures were determined by subtracting the false alarm rate for the average of weakly related/unpresented words and unrelated/ unpresented words from the false alarm rate for critical lures. The values are presented in Table 1 .

A correlational analysis examined whether individuals were more likely to false alarm to the critical lure when it was preceded at test by more rather than fewer related words. A small significant correlation was observed ( $r=$ $.29, p<.01)$. We had no particular expectation regarding this correlation, and we report it simply for comparison with Experiment 1, where the possibility of a test-based effect warranted further analysis. Consistent with Experiment 1 , the analysis dividing the test list into thirds showed no effect $\left[F(2,64)=1.29, M S_{\mathrm{e}}=0.21, p=.31\right]$.

Comparing the experiments. The two experiments, conducted separately, involved quite different response modes: keypress color identification versus vocal word reading. Although a comparison across experiments is not straightforward, it is worth doing. The two experiments were compared using hit rates for actually presented words and corrected false recognition rates for critical lures, to take account of the differences in false alarms to weakly related/unpresented words and unrelated/unpresented words between the two experiments. A $2 \times 2$ withinsubjects ANOVA with the factors of task/experiment (color identification vs. word reading) and word type (actually presented words and critical lures) yielded significant main effects of task/experiment $[F(1,33)=$ $\left.15.72, M S_{\mathrm{e}}=0.09, p<.001\right]$ and of word type $[F(1,33)=$ $\left.14.96, M S_{\mathrm{e}}=0.06, p<.001\right]$. Most notably, there was also a significant interaction between task/experiment and word type $\left[F(1,33)=4.91, M S_{\mathrm{e}}=0.06, p<.05\right]$. Whereas the hit rate for actually presented words increased dramatically between Experiments 1 and 2, the false alarm rate to critical lures was unchanged.

In support of this conclusion, planned comparisons across task/experiment indicated a significant difference in hit rates for actually presented DRM list words $[t(33)=$ $8.72, p<.001$ ], with word reading (Experiment 2 ) leading to much better recognition of actually presented words than did color naming (Experiment 1). In contrast, although the corrected false recognition of critical lures was numerically greater in Experiment 2 (.58) than in Experiment $1(.47)$, this difference was not significant $[t(33)=$ $1.26, p=.22$ ]. Thus, false alarms to critical lures occurred at a consistently high rate, despite the large difference in correct recognition for actually presented words. An analogous $d^{\prime}$ analysis supported the same conclusion. 


\section{GENERAL DISCUSSION}

Taken together, our two experiments demonstrate that false recognition of critical lures does not depend on intentional processing of DRM list words. Robust false memory was observed despite participants' not attempting to memorize (Experiments 1 and 2) or even to read (Experiment 1) presented words. In Experiment 1, the participants performed a color identification task, ignoring the DRM list words presented as distractors. As expected, on a subsequent surprise test, recognition of actually presented words was poor, although significantly above baseline (in comparison with false alarms to unpresented words). Whereas false alarms to weakly related/unpresented and unrelated/unpresented words were also low, false alarms to critical lures were high $(70 \%)$. Even after one takes other false alarms into account, the participants were virtually as likely to false alarm to a critical lure (47\%) as they were to correctly recognize an actually presented word (54\%). This is the first demonstration that false memory can be elicited without the intentional processing of DRM list words.

In Experiment 2, the participants read words rather than identifying their colors, but the recognition test was still a surprise. As expected, there was a dramatic increase in correct recognition of actually presented words and a decrease in false recognition of weakly related/ unpresented words and unrelated/unpresented words. False alarms to critical lures, however, remained robust. The slightly higher corrected false recognition rate for critical lures in Experiment 2 than in Experiment 1 was not reliable. Thus, false recognition of critical lures was unaffected by whether words were read intentionally or unintentionally, despite large changes in recognition for actually presented words.

Our results add to the literature concerning levels-ofprocessing effects on false memory. There has been debate regarding whether these manipulations affect true and false memory similarly (Rhodes \& Anastasi, 2000; Thapar \& McDermott, 2001; Toglia et al., 1999) or whether they have no effect on false memory (Read, 1996; Tussing \& Greene, 1997). Although not definitive-numerically higher false recognition did occur when DRM list words were read intentionally-the nonsignificant influence of intentionality on false recognition in our study suggests that levels-of-processing effects are greater on true than on false memories. We note, however, that most previous studies have compared "shallow" and "deep" processing, whereas our study may be seen as comparing "shallow" and "very shallow" processing, so it is still possible that levels-of-processing effects on false memory may be greater when deeper processing is engaged.

The present results are consistent with the activation/ monitoring approach (Roediger et al., 2001) wherein false memory can result from automatic activation of critical lures via spreading semantic activation. The critical lure may be activated frequently as successive DRM list words appear (given its association with each list word), perhaps explaining why critical lures were falsely recognized so often relative to actually presented words in Experiment 1. Even after one takes false alarms to other words into account, participants were as likely to correctly recognize an actually presented word as to falsely recognize a critical lure. Further evidence in favor of activation/monitoring has been reported by Seamon and his colleagues (Seamon et al., 1998; Seamon, Lee, et al., 2002; Seamon, Luo, Shulman, Toner, \& Caglar, 2002), although in their studies word reading was intentional and the memory test was expected.

Overall, the activation/monitoring approach provides a reasonable account of why false memories occur in the DRM paradigm. This approach is attractive because it assumes that false memories can occur either from conscious, elaborative processes, or from automatic spreading of activation to critical lures when DRM list words are processed. It would be difficult to reconcile our results with any theory of false memory that assumes that such memory errors result solely from conscious elaborative processes, given that robust false memory occurred in the absence of any attempt to read or memorize DRM list words.

\section{REFERENCES}

BARTLETt, F. C. (1932). Remembering: A study in experimental and social psychology. Cambridge: Cambridge University Press.

Brainerd, C. J., \& REYNA, V. F. (2002). Fuzzy-trace theory and false memory. Current Directions in Psychological Science, 11, 164-169.

Craik, F. I. M., \& LockHART, R. S. (1972). Levels of processing: A framework for memory research. Journal of Verbal Learning \& Verbal Behavior, 11, 671-684.

DEESE, J. (1959). On the prediction of occurrence of particular verbal intrusions in immediate recall. Journal of Experimental Psychology, 58, 17-22.

Graves, R. E., \& Bradley, R. (1991). Millisecond timing on the IBM $\mathrm{PC} / \mathrm{XT} / \mathrm{AT}$ and PS/2: A review of the options and corrections for the Graves and Bradley algorithm. Behavior Research Methods, Instruments, \& Computers, 23, 377-379.

MACLEOD, C. M. (1991). Half a century of research on the Stroop effect: An integrative review. Psychological Bulletin, 109, 163-203.

MACLEOD, C. M. (2000). Using the Stroop task as an indirect measure of memory. Paper presented at the XXVII International Congress of Psychology, Stockholm.

MACLEOD, C. M., \& DodD, M. D. (2001). Singling out the Stroop effect: What really happens when only one letter is colored? Paper presented at the South Carolina Bicentennial Symposium on Attention, Columbia, SC.

MCDERmott, K. B., \& WATson, J. M. (2001). The rise and fall of false recall: The impact of presentation duration. Journal of Memory \& Language, 45, 160-176.

READ, J. D. (1996). From a passing thought to a false memory in 2 minutes: Confusing real and illusory events. Psychonomic Bulletin \& Review, 3, 105-111.

Rhodes, M. G., \& Anastasi, J. S. (2000). The effects of a levels-ofprocessing manipulation on false recall. Psychonomic Bulletin \& Review, 7, 158-162.

Roediger, H. L., III, \& McDermott, K. B. (1995). Creating false memories: Remembering words not presented in lists. Journal of Experimental Psychology: Learning, Memory, \& Cognition, 21, 803-814.

RoEdiger, H. L., III, WATSON, J. M., MCDermott, K. B., \& GAllo, D. A. (2001). Factors that determine false recall: A multiple regression analysis. Psychonomic Bulletin \& Review, 8, 385-407. 
SeAmon, J. G., Lee, I. A., Toner, S. K., Wheeler, R. H., Goodkind, M. S., \& BIRCH, A. D. (2002). Thinking of critical words during study is unnecessary for false memory in the Deese, Roediger, and McDermott procedure. Psychological Science, 13, 526-531.

SeAmon, J. G., LuO, C. R., \& GALlo, D. A. (1998). Creating false memories of words with or without recognition of list items: Evidence for nonconscious processes. Psychological Science, 9, 20-26.

Seamon, J. G., Luo, C. R., Shulman, E. P., Toner, S. K., \& Caglar, S. (2002). False memories are hard to inhibit: Differential effects of directed forgetting on accurate and false recall in the DRM procedure. Memory, 10, 225-237.

Stadler, M. A., Roediger, H. L., III, \& McDermott, K. B. (1999). Norms for word lists that create false memories. Memory \& Cognition, 27, 494-500.

Stroop, J. R. (1935). Studies of interference in serial verbal reactions. Journal of Experimental Psychology, 18, 643-662.

ThAPAR, A., \& MCDERMOTT, K. B. (2001). False recall and false recognition induced by presentation of associated words: Effects of retention interval and level of processing. Memory \& Cognition, 29, 424-432.

Toglia, M. P., Neuschatz, J. S., \& Goodwin, K. A. (1999). Recall accuracy and illusory memories: When more is less. Memory, 7, 233-256.

Tussing, A. A., \& GREE NE, R. L. (1997). False recognition of associates: How robust is the effect? Psychonomic Bulletin \& Review, 4, 572-576.

UNDERWOOD, B. J. (1965). False recognition produced by implicit verbal responses. Journal of Experimental Psychology, 70, 122-129.

Williams, J. M. G., Mathews, A., \& MacLeod, C. (1996). The emotional Stroop task and psychopathology. Psychological Bulletin, 120, 3-24.

\section{NOTES}

1. The levels-of-processing manipulation also failed to influence hit rates in the Tussing and Greene (1997) study, which could suggest that the encoding manipulation did not work as expected.

2. Thapar and McDermott (2001) used a similar color-naming manipulation, although they explicitly informed their participants before the colornaming session that their memory for the presented words would be tested.

3. The reported experiment was actually the second experiment that participants engaged in during the session. The practice session preceded the first experiment, which was also a color identification task (part of another project) that used only nonwords and letter strings as distractors. This experimental order permitted participants to become quite proficient at the buttonpressing procedure and helped to foster the belief that the present experiment was merely a continuation of the color identification task that preceded it.

4. Corrected false recognition rates for critical lures were actually calculated three ways in each experiment: once using false alarms to weakly related/unpresented words as the baseline; once using false alarms to unrelated/unpresented words as the baseline, and once using the average of false alarms to weakly related/unpresented words and unrelated/unpresented words as the baseline. Although these different baselines yielded small numerical differences in corrected false recognition for critical lures, no statistically significant difference emerged regardless of the baseline used.

(Manuscript received February 4, 2002; revision accepted for publication January 13, 2003.) 